\title{
Estudo radiográfico em pacientes submetidos à artroplastia total de joelho não cimentada: Segmento mínimo de 2 anos* $^{*}$
}

\section{Radiographic Study in Patients Submitted to Cementless Total Knee Arthroplasties: Minimum Follow-Up of 2 Years}

\author{
Sérgio Mainine ${ }^{1}$ José Antônio Marinho ${ }^{1}$ Bruna Gabriela Passarini de Oliveira ${ }^{20}$ \\ Enrico Montorsi Zanon ${ }^{1}$ Bruno Machado Pereira ${ }^{1}$ Rafael Fonseca Rodrigues de Souza ${ }^{1}$
}

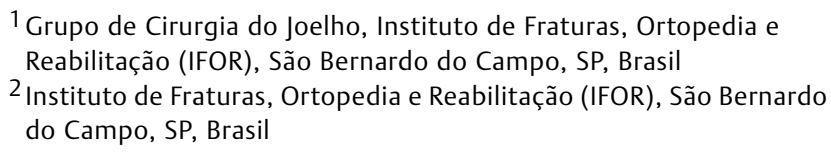

2 Instituto de Fraturas, Ortopedia e Reabilitação (IFOR), São Bernardo do Campo, SP, Brasil

\begin{abstract}
Endereço para correspondência Sergio Mainine, Chefe de Equipe da Cirurgia do Joelho. Rua Bela Vista, 426. São Bernardo do Campo, SP, Brasil. CEP 09715-030 (e-mail: sergiomainine@gmail.com).
\end{abstract}

Rev Bras Ortop 2021;56(5):628-633.

\section{Resumo \\ Palavras-chave \\ - artroplastia do joelho \\ - artrose \\ - prótese do joelho \\ - cimentação}

Objetivo Avaliar radiograficamente a existência de sinais de soltura precoce das artroplastias totais de joelho não cimentadas Score (Amplitude Surgical SAS, Valence, France), verificadas com um tempo de seguimento que variou de 2 a 5,75 anos (média de 3,75 anos).

Métodos Investigação longitudinal descritiva de caráter observacional, não comparativa, realizada através de estudo radiográfico estático de controle anual, de uma série de casos, em um único centro, todos operados pelo mesmo cirurgião (S.M.). Foram incluídas todas as artroplastias não cimentadas que se enquadraram nos critérios de inclusão e exclusão realizadas no período de março de 2012 a outubro de 2014 .

Resultados Dentre as 46 artroplastias de joelho não cimentadas avaliadas em 40 pacientes, não foram verificados sinais radiográficos de soltura precoce.

Conclusão A artroplastia não cimentada promove ótima osteointegração, não havendo soltura precoce, sendo fundamental que a técnica cirúrgica seja perfeitamente respeitada.

\footnotetext{
Trabalho realizado no Serviço de Cirurgia do Joelho do Instituto de Fraturas, Ortopedia e Reabilitação (IFOR), São Bernardo do Campo, SP, Brasil.
}

recebido

03 de Abril de 2020

aceito

01 de Dezembro de 2020
DOI https://doi.org/

10.1055/s-0041-1731355. ISSN $0102-3616$

\author{
(c) 2021. Sociedade Brasileira de Ortopedia e Traumatologia. All \\ rights reserved. \\ This is an open access article published by Thieme under the terms of the \\ Creative Commons Attribution-NonDerivative-NonCommercial-License, \\ permitting copying and reproduction so long as the original work is given \\ appropriate credit. Contents may not be used for commercial purposes, or \\ adapted, remixed, transformed or built upon. (https://creativecommons.org/ \\ licenses/by-nc-nd/4.0/) \\ Thieme Revinter Publicações Ltda., Rua do Matoso 170, Rio de \\ Janeiro, RJ, CEP 20270-135, Brazil
}


Abstract

\section{Keywords}

- arthroplasty, replacement, knee

- arthrosis

- knee prosthesis

- cementation
Objective Radiographic evaluate if there are signs of early loosening of the cementless total knee arthroplasties Amplitude-Score ${ }^{\circledR}$ (Amplitude Surgical SAS, Valence, France), checking with a follow-up time ranging from 2 to 5.75 years (mean of 3.75 years).

Methods Descriptive longitudinal investigation of observational nature, non-comparative, through a static radiographic study of annual control, of a case series, in a single center, all operated on by the same surgeon (S.M.). All cementless arthroplasties that met the inclusion and exclusion criteria performed from March 2012 to October 2014 were included.

Results Among the 46 cementless knee arthroplasties evaluated in 40 patients, no radiographic signs of early loosening were verified.

Conclusion Cementless arthroplasty promotes optimal osteointegration, with no early release, and it is essential that the surgical technique is perfectly respected.

\section{Introdução}

A osteoartrose $(\mathrm{OA})$ é uma doença articular degenerativa que evolui com processo inflamatório crônico, causando degeneração articular. Caracteriza-se por dor, rigidez matinal, crepitação, atrofia muscular e, no que tange aos aspectos radiográficos, observa-se estreitamento do espaço articular, formações de osteófitos, esclerose do osso subcondral e formações císticas. ${ }^{1}$

A etiologia pode ser idiopática ou pós-traumática. A póstraumática pode acometer qualquer idade. A idiopática, com fortes indícios genéticos, acomete principalmente indivíduos mais idosos, embora também ocorra em adultos de meia idade, em especial mulheres, entre a $5^{a}$ e a $6^{a}$ décadas de vida e no período pós-menopausa. É interessante que, abaixo dos 40 anos, sua ocorrência é praticamente igual para ambos os sexos. Estima-se que a maioria da população acima dos 65 anos irá sofrer com algum grau de OA, que é, no envelhecimento, a principal causa de incapacitação funcional quando comparada a qualquer outra enfermidade. E nessa faixa etária, cerca de $50 \%$ apresentará artrose no joelho. ${ }^{1,2}$

Na falha do tratamento conservador, a artroplastia total do joelho (ATJ) tem sido bem estabelecida restaurando a função, aliviando a dor e corrigindo as deformidades e instabilidades, tornando-se uma opção mesmo para pacientes mais jovens com artrite grave de joelho. ${ }^{1}$

As taxas anuais de ATJ estão aumentando progressivamente em razão da longevidade da população, atrelado ao fato de que cirurgias vem sendo realizadas em pacientes cada vez mais jovens. Um importante estudo mostrou que as revisões de ATJ nos Estados Unidos duplicaram no ano de 2015 e terão aumento da ordem de $600 \%$ até o ano de $2030 .^{2}$

Pacientes mais jovens, com maior expectativa de vida, nível mais alto de atividade e exigência do implante, requerem uma fixação segura para garantir a longevidade dos componentes e a redução das revisões. Contudo, a utilização de fixação cimentada, embora demonstre excelentes resultados, tem sido reexaminada. ${ }^{3,4}$

A fixação ideal de uma ATJ ainda é debatida. A principal questão é se o uso de cimento é mais eficiente do que a fixação press-fit em termos de assegurar a estabilidade durável. $\mathrm{O}$ uso de cimento em ATJ tem sido associado com excelentes resultados clínicos e baixos índices de soltura asséptica em longo prazo de seguimento, sendo o método mais comum de fixação. No entanto, alterações da interface cimento-osso, considerada zona crítica de estresse e soltura, fomentaram a busca de novos métodos de fixação dos componentes. $^{5-8}$

Os potenciais benefícios da fixação não cimentada incluem a preservação do osso, menor tempo de cirurgia, facilidade de revisão e eliminação de complicações associadas à fixação cimentada, incluindo desgaste e retenção de fragmentos soltos de cimento, extrusão do mesmo, resposta biológica ao polimetilmetacrilato, trombose venosa profunda, choque, além de necrose térmica devido à sua polimerização. Por fim, as revisões de artroplastias cimentadas são tecnicamente mais complicadas em relação aos implantes não cimentados, particularmente por conta da perda óssea frequente após a remoção dos componentes e cimento residual. ${ }^{9-11}$

A fixação cimentada é conhecida por proporcionar uma boa fixação inicial e não migrar no período pós-operatório imediato, embora possa apresentar micro movimento em 60 meses. Já a interface não cimentada pode migrar mais cedo, ou seja, nos primeiros 3 meses de pós-operatório, geralmente atingindo estabilidade após esse intervalo; porém, após a osteointegração entre o osso e o metal, há formação de uma ligação biológica que tende a proporcionar melhores resultados em longo prazo. ${ }^{12,13}$ A soltura tibial precoce ainda é o principal problema apontado pelos críticos da técnica sem cimento.

O principal parâmetro para avaliar a soltura dos componentes das artroplastias é a presença de linhas de radioluscência nas radiografias pós-operatórias, situadas na periferia dos componentes descritos pela The Knee Society ${ }^{14}$ (-Figuras 1 e 2). Sendo significantes radioluscências com mais de $1 \mathrm{~mm}$ de aumento da sua largura observado na evolução e migração dos componentes. As linhas de radioluscência podem estar presentes desde o pós-operatório imediato e só se tornarão uma preocupação quando houver 


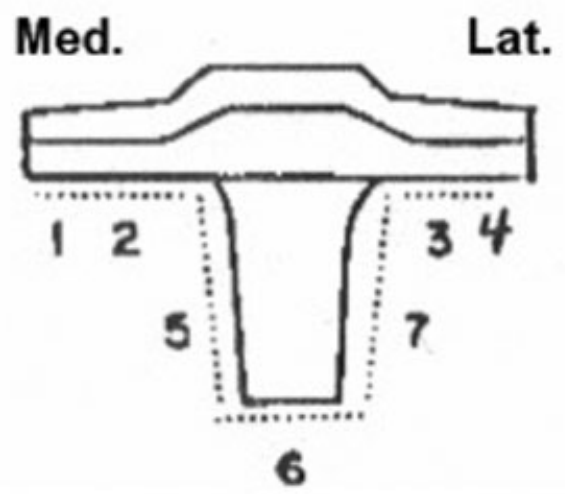

Fig. 1 Zonas ao redor dos componentes da prótese onde deve-se observar radioluscências no componente tibial. 1 e 2 - platô medial; 3 e 4 - platô lateral; 5, 6 e 7 - ao redor da haste.

modificação de seu padrão ao longo do acompanhamento, o que pode significar a soltura dos componentes e a necessidade de revisão da artroplastia.

Desse modo, neste estudo, objetivou-se avaliar em curto prazo se houve soltura precoce de componentes da prótese de joelho não cimentada Score (Amplitude Surgical SAS, Valence, France).

\section{Casuística e Método}

O trabalho foi submetido para avaliação do comitê de ética em pesquisa desta instituição e aprovado para execução conforme o número certificado de apresentação de apreciação ética (CAAE) - 27786119.2.0000.5625, parecer: 3.814.869.

Este é um estudo longitudinal descritivo de caráter observacional, não comparativo, de série de casos, em um único centro, todos operados pelo mesmo cirurgião (S. M.), avaliando soltura precoce dos componentes de próteses não cimentadas através de estudo radiográfico estático de controle anual.

Utilizou-se como critérios de inclusão:

- Artroplastia total não cimentada;

- Índice de massa corporal (IMC) $<35 \mathrm{~kg} / \mathrm{m}^{2}$;

- Tempo de seguimento pós-operatório mínimo de 2 anos;

- Ter lido e assinado o termo de consentimento livre e esclarecido (formulado obedecendo às recomendações da Resolução $n^{\circ} 466$ de 12 de dezembro de 2012 do Conselho Nacional de Saúde), declarando ter compreendido todas as explicações e ter concordado plenamente com a pesquisa.

Excluindo-se pacientes:

- Que tiveram artroplastia anterior a esta cirurgia;

- Doenças metabólicas e reumatológicas;

- Infecção ou malignidade ativa.

Foram incluídas todas as artroplastias não cimentadas que se enquadraram nos critérios de inclusão e exclusão realizadas no período de março de 2012 a outubro de 2014.

Todas as cirurgias foram realizadas através de via de acesso parapatelar medial, sacrificando o ligamento cruzado posterior utilizando prótese total de joelho não cimentada de

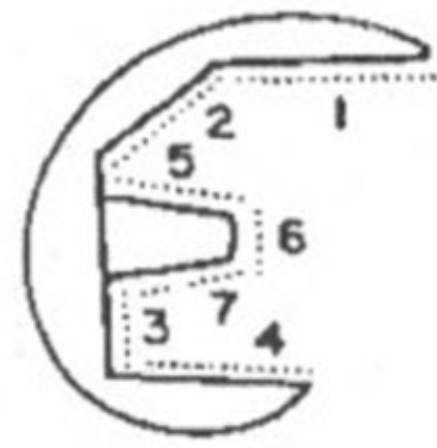

Fig. 2 Zonas ao redor dos componentes da prótese onde deve-se observar radioluscências no componente femoral. 1 e 2 - região anterior; 3 e 4 - área posterior; 5, 6 e 7 - haste e porção central.

plataforma rotatória Score, com estabilização ultra-congruente, em que os componentes femorais e tibiais são de cromo-cobalto com duplo revestimento de titânio e revestimento poroso de 80 micrômetros de hidroxiapatita. 0 componente patelar de polietileno, em cúpula, com três pinos de fixação, cimentado, só não foi utilizado em dois pacientes, três joelhos. Foi colocado dreno a vácuo em todos os casos, sendo retirado quando o débito era menor que $50 \mathrm{ml}$ em 24 horas, não ultrapassando 48 horas, independente do débito.

Os pacientes receberam alta hospitalar no $3^{\circ}$ dia pósoperatório, permitindo carga total com auxílio de andador para equilíbrio e iniciando fisioterapia ambulatorial para ganho de amplitude de movimentos. Foram avaliados com 1, 3, 6 e 12 e 24 semanas. Após esta fase inicial, tiveram retorno ambulatorial a cada 6 meses até 1 ano, quando foi realizado controle radiográfico de rotina.

As radiografias do joelho operado, na posição de frente ortostática e em perfil absoluto do joelho com 30 graus de flexão, foram analisadas por 2 cirurgiões com experiência em artroplastia, cegos entre si, com ênfase no padrão de possíveis linhas de radioluscência no fêmur e na tíbia, utilizando os parâmetros da The Knee Society para avaliação dos implantes e progressão da radioluscência ${ }^{15}$ (- Tabela 1; - Figuras 1 e 2 ).

\section{Resultados}

Foram avaliadas 46 artroplastias em 40 pacientes ( 6 bilaterais) não cimentadas, com plataforma rotatória Score, realizadas no período de março de 2012 a outubro de 2014 . As características dessa população são apresentadas na - Tabela 2.

$\mathrm{Na}$ análise das radiografias, não encontramos linhas de radioluscência ao redor do fêmur. Na análise do componente

Tabela 1 Interpretação das imagens nas radiografias

\begin{tabular}{|l|l|}
\hline Linha de radioluscência & Interpretação \\
\hline$\leq 0,4 \mathrm{~mm}$ & Normal \\
\hline De $0,5-0,9 \mathrm{~mm}$ & $\begin{array}{l}\text { Acompanhar a cada } 3 \text { meses } \\
\text { se há progressão }\end{array}$ \\
\hline$\geq 1 \mathrm{~mm}$ & Possível ou iminente falha \\
\hline
\end{tabular}


Tabela 2 Distribuição da população estudada

\begin{tabular}{|l|l|l|l|l|}
\hline & Idade (anos) & Sexo & Lado & Tempo de seguimento \\
\hline 40 pacientes & Mínima: 46,9 & F: 30 & D: 26 & Mínimo: 2 anos \\
\hline 46 artroplastias & Máxima: 83,0 & M: 10 & E: 20 & Máximo: 5,75 anos \\
\hline ( 6 bilaterais) & Média: 60,8 & & Médio: 3,75 anos \\
\hline
\end{tabular}

Abreviaturas: D, direito; E, esquerdo; F, feminino; M, masculino.

Tabela 3 Resultados da análise das radiografias realizadas na última visita dos pacientes

\begin{tabular}{|l|l|l|l|}
\hline & $\begin{array}{l}\text { Linhas de } \\
\text { radioluscência } \\
(\mathrm{AP})\end{array}$ & $\begin{array}{l}\text { Linhas de } \\
\text { radioluscência } \\
(\mathrm{P})\end{array}$ & Total \\
\hline Fêmur & 0 & 0 & 0 \\
\hline Tíbia & $5(10,8 \%)$ & $1(2,2 \%)$ & $6(13,0 \%)$ \\
\hline
\end{tabular}

Abreviaturas: AP, anteroposterior; P, perfil.

tibial, foram observadas linhas de radioluscência em 6 (13,0\%) artroplastias, sendo $5(10,8 \%)$ na visão ânteroposterior $(\mathrm{AP})$ e $1(2,2 \%)$ na visão em perfil $(\mathrm{P})$, ocorrendo principalmente nas zonas 1 e 4 , com espessura média de $1 \mathrm{~mm}$, presentes desde o início e não progressivas (-Tabela $\mathbf{3}$, -Figuras 1-3).

Portanto, nenhum caso apresentou suspeita de soltura ou instabilidade dos componentes e nenhuma revisão de artroplastia devido à falha mecânica foi realizada.

Houve necessidade de revisão por infecção, e não por soltura da prótese, após 7 meses, em um paciente com válvula cardíaca em uso de anticoagulante oral e descontrole da coagulação, evoluindo com hemartroses de repetição e infecção hematogênica, pois em momento algum foi realizada punção articular. Após insucesso com limpeza e conservação do implante, optou-se pela sua retirada, quando foi observada ótima osteointegração dos componentes femoral e tibial, estando, portanto, fixos (-Figura 4 e 5 ).

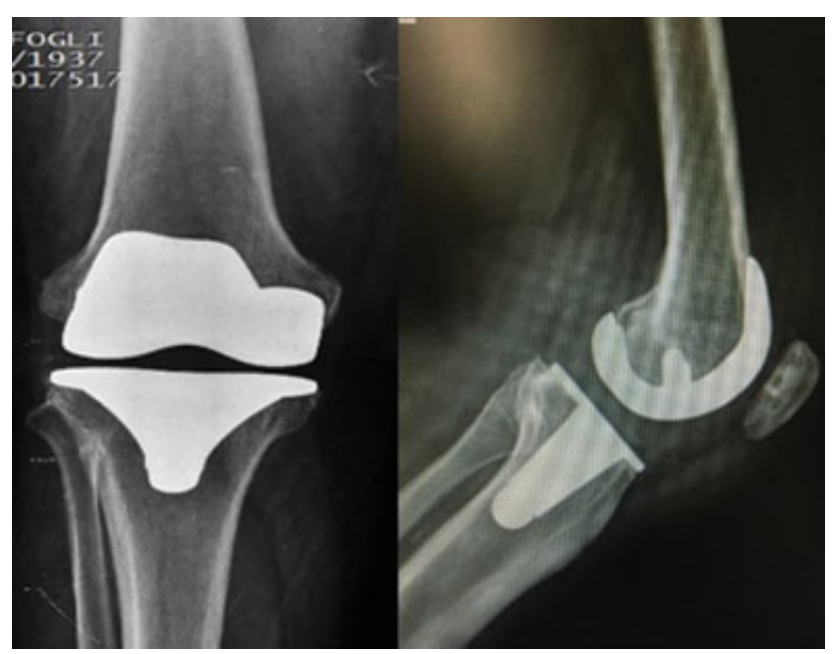

Fig. 3 Radiografias demostrando prótese total do joelho sem cimentação e sem linhas radioluscentes ao redor dos componentes.

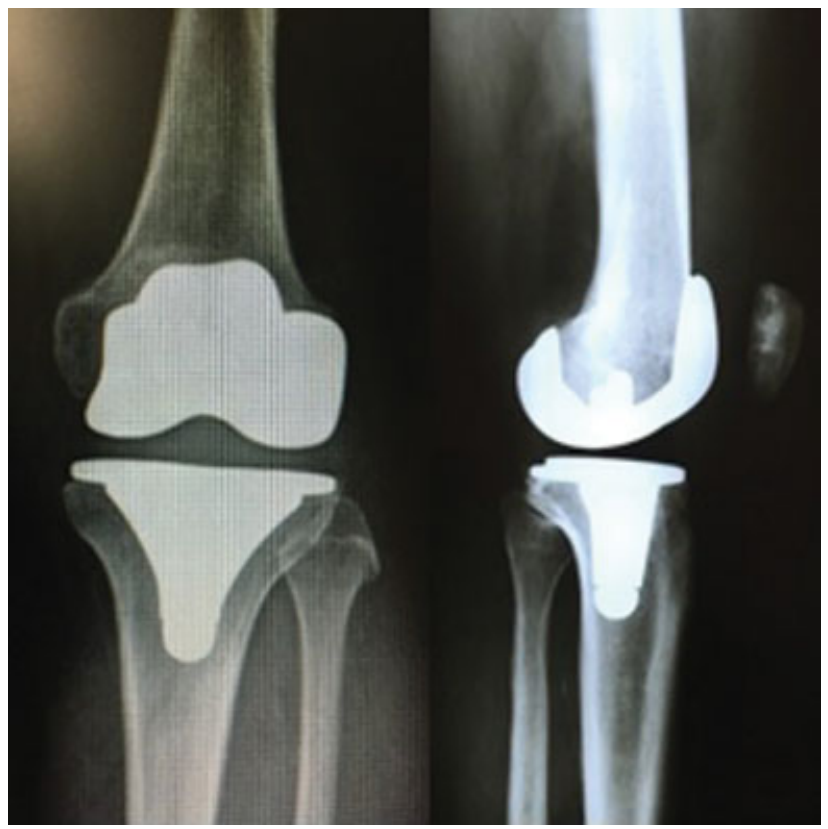

Fig. 4 Componentes da prótese do paciente $n^{\circ} 20$, que foi retirada devido a infecção. Observa-se integração óssea nas superfícies porosas da prótese.

Outro paciente, com 3 anos de pós-operatório, sofreu trauma com fratura femoral periprotética, e foi submetido à redução e fixação interna com placa bloqueada, mantendo a fixação do componente e boa evolução (- Figura 6).

\section{Discussão}

O estudo da fixação sem cimento na ATJ começou a ser utilizado em meados dos anos 80 depois de estudos laboratoriais mostrarem que o crescimento ósseo para a estabilidade da interface implante-osso interfere na durabilidade do componente fixado. ${ }^{16,17}$

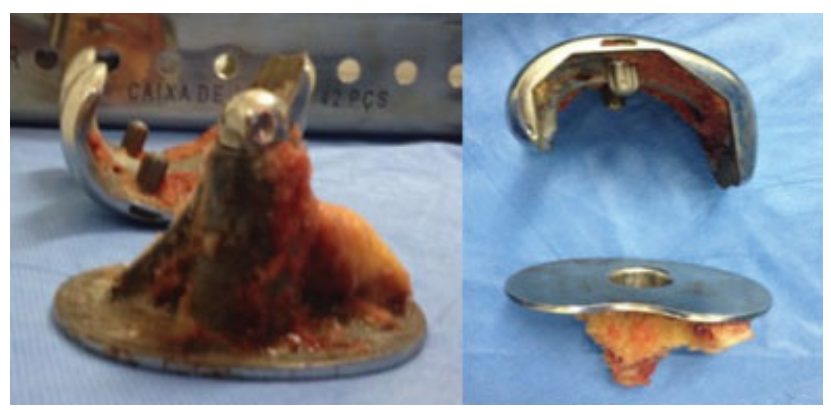

Fig. 5 Radiografia da paciente $n^{\circ} 22$, demonstrando prótese total do joelho esquerdo e osteossíntese no fêmur ipsilateral. 


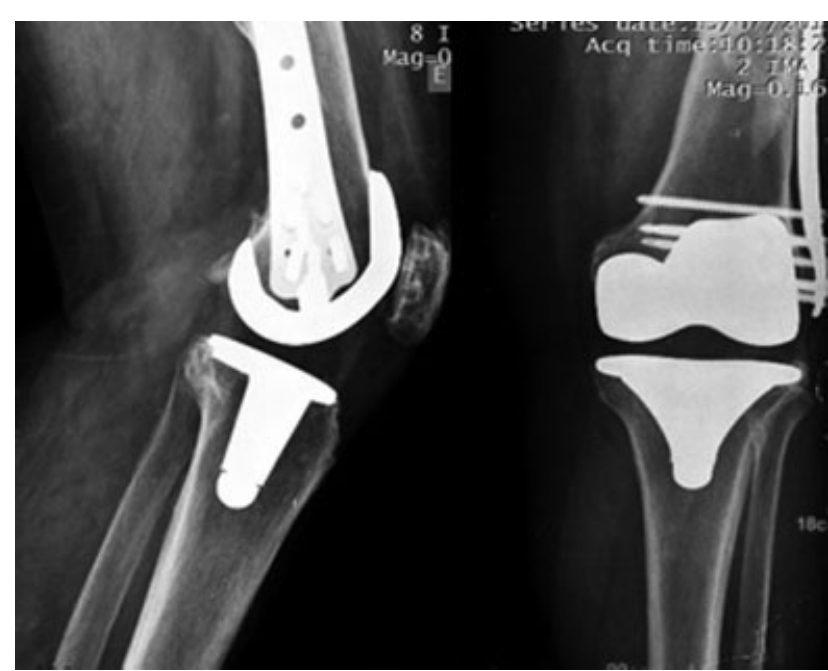

Fig. 6 Demonstrando discreta linha de radioluscência ao redor do componente tibial da prótese, que não foi progressiva, portanto não constitui caso de soltura do implante.

Em estudos que analisaram a migração de implantes sem cimento, foi demonstrado haver algum grau de migração dos componentes nos primeiros 6 meses até que ocorra a osteointegração e sua estabilização. Já os implantes cimentados apresentam migração inicial menor, porém constante ao longo de 5 anos. $^{12}$

A presença de radioluscência ao redor do implante sem cimento não significa necessariamente a soltura do componente (-Figura 6), sendo possivelmente decorrente da migração inicial do mesmo, podendo não apresentar soltura dos implantes, ${ }^{13}$ ou mesmo radioluscência em região de osso ebúrneo presente antes da cirurgia, que acontece tanto nas próteses cimentadas como nas não cimentadas. ${ }^{18}$

Existem inúmeros parâmetros de avaliação e quantificação das linhas de radioluscência e da soltura dos componentes da artroplastia. $\mathrm{O}$ mais utilizado atualmente é a classificação da The Knee Society (Sociedade do Joelho americana) baseada em radiografias do joelho de frente e perfil, em que as linhas de radioluscência são quantificadas de acordo com sua localização ao redor dos componentes tibial e femoral. ${ }^{14}$ Geralmente, esta avaliação é realizada por cirurgiões experientes que determinam se os componentes são estáveis ou instáveis. Estudos com radiostereografia ou radiografias dinâmicas podem sensibilizar o diagnóstico de instabilidade e soltura dos componentes da prótese. ${ }^{10,11,13,15,19,20}$

Na literatura encontra-se uma média de $1.4 \mathrm{~mm}$ de radioluscência no fêmur, principalmente nos segmentos 1 e 2 , em seguimentos de longo prazo. Não observamos essas linhas no fêmur nos nossos casos avaliados em curto período pós-operatório. Já na tíbia, a literatura mostra essas linhas radioluscentes de 1,4 mm, principalmente nas zonas 1 e 4 , ou seja, na periferia medial e lateral do implante, num seguimento médio de 10 anos. Esse resultado é semelhante ao encontrado em nossa série, sendo que obtivemos uma média de $1 \mathrm{~mm}$ nas mesmas localizações.

Ao longo de décadas, estudos in vitro demonstraram que a utilização de plataformas rotatórias em ATJs não cimentadas estão associadas com um melhor desempenho fisiológico e sobrevivência do implante, bem como com a redução das tensões na interface osso-metal. Vários estudos no cenário clínico também mostraram sobrevivência em longo prazo de press-fit com plataformas rotativas, com aumento de $83 \%$ para $99,4 \%{ }^{21}$ Em nossa série, usamos em todos os casos a prótese Score não cimentada com plataforma rotatória. Não temos experiência para compará-la com próteses sem cimento com plataforma fixa.

Para uma osteointegração adequada, é fundamental que os cortes ósseos sejam muito precisos e a prótese de teste apresente um ótimo press-fit. Caso isso não ocorra, é melhor mudar o planejamento e colocar a prótese cimentada. Por isso é importante dispor dos dois tipos de implante na sala de cirurgia.

Por fim, outro ponto importante para bons resultados nas artroplastias sem cimento é o revestimento bioativo dos componentes, principalmente o componente tibial, proporcionando menos migração. Os estudos de análise radiostereográfica têm mostrado resultados diferentes quando se avalia componentes tibiais não cimentados revestidos com hidroxiapatita em comparação com componentes tibiais cimentados. $^{10} \mathrm{Em}$ um estudo prospectivo de pacientes jovens e ativos, Tai e Cross avaliaram 118 joelhos com implantes sem cimento revestidos com hidroxiapatita (Ativo; DJ Ortho, Sydney, Austrália) por um período de 5 a 12 anos. ${ }^{16}$ Duas revisões da tíbia por soltura asséptica e troca de um polietileno foram realizadas. A taxa de sobrevivência cumulativa foi $92,1 \%$ (incluindo o caso da troca de polietileno). ${ }^{14}$

Apesar da artroplastia sem cimento do joelho ter sido desenvolvida voltada para jovens com maior nível de atividade física, estudos recentes mostraram resultados semelhantes quanto à sobrevida deste tipo de implante na população acima dos 75 anos. $^{22} \mathrm{E}$, em pacientes obesos mórbidos, os resultados foram melhores do que os das cirurgias com implantes cimentados. ${ }^{23}$

Portanto, faz-se necessário aprofundar o estudo nessa linha de artroplastias, a qual pode ser uma tendência nas próteses de joelho, sobretudo em pacientes jovens, ativos, com alta demanda, com possibilidade de estender essa indicação num futuro próximo.

\section{Conclusão}

Não obstante, a geração atual de implantes sem cimento apresenta resultados excelentes não somente em relação ao press-fit, mas também em proporcionar estabilidade inicial aos componentes e permitir uma interface implante-osso mais biológica.

Apesar das limitações do nosso estudo - curto tempo de seguimento, não-randomização da amostra e o fato de um único cirurgião ter realizado todas as cirurgias - o ponto relevante evidenciado no estudo foi a ausência de soltura das próteses no período acompanhado.

\section{Suporte Financeiro}

Não houve suporte financeiro de fontes públicas, comerciais, ou sem fins lucrativos. 


\section{Conflito de Interesses}

Os autores declaram não haver conflito de interesses.

\section{Referências}

1 Silva PMGO. Prótese total do joelho - a história da arte: revisão bibliográfica [dissertação]. Covilhã: Universidade da Beira Interior; 2010

2 Kurtz S, Ong K, Lau E, Mowat F, Halpern M. Projections of primary and revision hip and knee arthroplasty in the United States from 2005 to 2030. J Bone Joint Surg Am 2007;89(04): 780-785

3 Harwin SF, Elmallah RK, Jauregui JJ, Cherian JJ, Mont MA. Outcomes of a newer-generation cementless total knee arthroplasty design. Orthopedics 2015;38(10):620-624

4 Harwin SF, Kester MA, Malkani AL, Manley MT. Excellent fixation achieved with cementless posteriorly stabilized total knee arthroplasty. J Arthroplasty 2013;28(01):7-13

5 Lombardi AV Jr, Berasi CC Jr, Berend KR. Evolution of tibial fixation in total knee arthroplasty. J Arthroplasty 2007;22(04, Suppl 1):25-29

6 Naudie DD, Ammeen DJ, Engh GA, Rorabeck CH. Wear and osteolysis around total knee arthroplasty. J Am Acad Orthop Surg 2007;15(01):53-64

7 O'Rourke MR, Callaghan JJ, Goetz DD, Sullivan PM, Johnston RC. Osteolysis associated with a cemented modular posterior-cruciate-substituting total knee design : five to eight-year follow-up. J Bone Joint Surg Am 2002;84(08):1362-1371

8 Noble PC, Conditt MA, Thompson MT, et al. Extraarticular abrasive wear in cemented and cementless total knee arthroplasty. Clin Orthop Relat Res 2003;(416):120-128

9 Bert JM, McShane M. Is it necessary to cement the tibial stem in cemented total knee arthroplasty? Clin Orthop Relat Res 1998; (356):73-78

10 Clarke MT, Green JS, Harper WM, Gregg PJ. Cement as a risk factor for deep-vein thrombosis. Comparison of cemented TKR, uncemented TKR and cemented THR. J Bone Joint Surg Br 1998;80(04): 611-613

11 Nilsson KG, Kärrholm J, Carlsson L, Dalén T. Hydroxyapatite coating versus cemented fixation of the tibial component in total knee arthroplasty: prospective randomized comparison of hydroxyapatite-coated and cemented tibial components with 5-year follow-up using radiostereometry. J Arthroplasty 1999;14(01): 9-20

12 Gao F, Henricson A, Nilsson KG. Cemented versus uncemented fixation of the femoral component of the NexGen CR total knee replacement in patients younger than 60 years: a prospective randomised controlled RSA study. Knee 2009;16(03):200-206

13 Nakama GY, Peccin MS, Almeida GJ, Lira Neto OdeA, Queiroz AA, Navarro RD. Cemented, cementless or hybrid fixation options in total knee arthroplasty for osteoarthritis and other non-traumatic diseases. Cochrane Database Syst Rev 2012;10(10):CD006193

14 Aebli N, Krebs J, Schwenke D, Hii T, Wehrli U. Progression of radiolucent lines in cementless twin-bearing low-contact-stress knee prostheses: a retrospective study. J Arthroplasty 2004;19 (06):783-789

15 Ewald FC. The Knee Society total knee arthroplasty roentgenographic evaluation and scoring system. Clin Orthop Relat Res 1989;(248):9-12

16 Tai CC, Cross MJ. Five- to 12-year follow-up of a hydroxyapatitecoated, cementless total knee replacement in young, active patients. J Bone Joint Surg Br 2006;88(09):1158-1163

17 Hungerford DS, Kenna RV, Krackow KA. The porous-coated anatomic total knee. Orthop Clin North Am 1982;13(01):103-122

18 Rorabeck CH, Bourne RB, Lewis PL, Nott L. The Miller-Galante knee prosthesis for the treatment of osteoarthrosis. A comparison of the results of partial fixation with cement and fixation without any cement. J Bone Joint Surg Am 1993;75(03):402-408

19 Regnér L, Carlsson L, Kärrholm J, Herberts P. Ceramic coating improves tibial component fixation in total knee arthroplasty. J Arthroplasty 1998;13(08):882-889

20 Tírico LEP, Pasqualin T, Pécora JO, Gobbi RG, Pécora JR, Demange MK. Study on implant stability in cementless total knee arthroplasty. Acta Ortop Bras 2012;20(04):230-234

21 Brown TE, Harper BL, Bjorgul K. Comparison of cemented and uncemented fixation in total knee arthroplasty. Orthopedics 2013;36(05):380-387

22 Bagsby DT, Issa K, Smith LS, et al. Cemented vs Cementless Total Knee Arthroplasty in Morbidly Obese Patients. J Arthroplasty 2016;31(08):1727-1731

23 Stähelin T, Kessler O, Pfirrmann C, Jacob HA, Romero J. Fluoroscopically assisted stress radiography for varus-valgus stability assessment in flexion after total knee arthroplasty. J Arthroplasty 2003;18(04):513-515 